\title{
Many-body effects in the Coulomb drag between low density electron layers
}

\author{
A. Yurtsever ${ }^{\mathrm{a}}$, V. Moldoveanu ${ }^{\mathrm{b}}$, B. Tanatar ${ }^{\mathrm{a}, *}$ \\ ${ }^{a}$ Department of Physics, Bilkent University, Bilkent, 06533 Ankara, Turkey \\ ${ }^{\mathrm{b}}$ National Institute of Materials Physics, P.O. Box MG-7, Bucharest-Magurele, Romania
}

Received 28 October 2002; received in revised form 5 November 2002; accepted 23 January 2003 by A.H. MacDonald

\begin{abstract}
Recent Coulomb drag experiments in low-density double-layer electron systems have the power of distinguishing various many-body formulations of the effective interactions. In this work we theoretically study the correlation effects on the drag resistivity in these systems within various models. The effective inter-layer interactions are best described by the generalization to the double-layer case of the Kukkonen-Overhauser approach which differs significantly from the self-consistent field approach of Singwi et al. [Phys. Rev. 176 (1968) 589]. Following the formulation of Vignale and Singwi [Phys. Rev. B 32 (1985) 2156] we derive an expression for the effective inter-layer interaction which embodies the many-body correlations through the local-field corrections. The drag resistivity is calculated within this approach together with the Hubbard approximation for the intra-layer local-field factor and a simple model for the inter-layer correlations. Comparison with the recent measurements of Kellogg et al. [Solid State Commun. 123 (2002) 515] yields very good agreement. Our results are also contrasted with the corresponding drag resistivities given by the Singwi et al. theory, the dynamic random-phase approximation and the Hubbard approximation. The significant differences found between these theories emphasize the strong sensitivity of the drag resistivity to the effective inter-layer interactions.
\end{abstract}

(c) 2003 Elsevier Science Ltd. All rights reserved.

PACS: 71.10.Ca; 73.20. - c; 73.20. - r; 71.45.Km

Keywords: A. Two-dimensional electron systems; D. Drag resistivity; D. Effective Coulomb interactions

The double-layer electron systems with and without perpendicular magnetic field attract lot of attention because of the large number of interesting physical effects they reveal [1]. One notable situation is the so-called drag effect when a current driven in one layer gives rise to a voltage difference in the second one [2]. The resulting drag resistivity $\rho_{\mathrm{D}}$ is directly proportional to the rate of momentum transfer between the layers, and therefore amenable to theoretical modeling.

Starting with the pioneering work of Gramila et al. [3] there has been a number of experimental investigations of the drag effect probing the various regimes of the parameter

\footnotetext{
* Corresponding author. Tel.: +90-312-290-1591; fax: +90-312266-4579.

E-mail address: tanatar@fen.bilkent.edu.tr (B. Tanatar).
}

space of layer densities, temperature, and separation distance between the quantum wells [4]. The experiments of Hill et al. [5] showed the evidence of plasmon enhancement to the drag resistivity at temperatures close to the Fermi temperature. They have also found significant differences with the random-phase approximation (RPA) based calculations [6] and the observed results. Noh et al. [7] performed similar experiments and argued that correlation induced multi-particle excitations must be included to account for the observed density dependences.

The theoretical efforts to account for the presumed correlation effects concentrated on improving upon the RPA. A commonly used treatment in this vein is the selfconsistent field approach of Singwi et al. [8] (STLS) which treats the short-range correlations effects better than RPA. Świerkowski et al. [9] applied the STLS formalism to 
double-layer electron-electron and electron-hole systems to demonstrate that the correlation effects enhance the drag resistivity by an order of magnitude compared to the RPA results. These calculations were compared with the experimental data of Gramila et al. [5] which were at relatively high density, viz. $n=1.5 \times 10^{11} \mathrm{~cm}^{-2}$.

With the advances in semiconductor growth techniques, very high mobility samples are routinely being manufactured to allow for measurements at low carrier densities. Thus, experimental results can be used as a testing ground for various many-body theories in the regime beyond weak coupling. The interaction effects or the coupling strength is described by the dimensionless parameter $r_{\mathrm{s}}=a / a_{\mathrm{B}}$ where $a$ and $a_{\mathrm{B}}$ are the average inter-particle distance and effective Bohr radius, respectively. The RPA breaks down for $r_{\mathrm{s}}>1$, and a more accurate theoretical model to describe the interaction effects in low-density electron systems is needed to account for drag resistivity measurements. This question has been raised in the experimental reports trying to identify the processes contributing to the observed drag effect.

Recent experiments on low density electron-electron [10] and hole-hole [11] double-layer systems revealed some new aspects. First, both papers reported a strong enhancement of the drag resistivity (up to 200 times larger in the case of hole-hole systems) than the values predicted by the theory). Second, at low temperatures $\rho_{\mathrm{D}}$ shows a stronger than $T^{2}$ dependence. Considering the hole-hole bilayer system [11] Hwang et al. [12] used the Hubbard approximation to account for the correlation effects together with several additional arguments to explain the experimental results. The experiments of Kellogg et al. [10] which were the starting point of our work have a two-fold importance: first the layers they used are separated by a distance $(280 \AA)$ which is smaller than the ones reported in previous experiments $[3,7]$ thus making the double-layer system more sensitive to interlayer interactions and second, one has $k_{\mathrm{F}} d \approx 1$ ( $d$ being the interlayer center to center separation), a regime where the $2 k_{\mathrm{F}}$ electron-electron backward scattering cannot be neglected. Both inter-layer interactions and $2 k_{\mathrm{F}}$ processes are expected to contribute to the drag resistivity and thus explain the discrepancies between the measured drag resistivity and the prediction given by a model [6] in which the static screening is given by a Thomas-Fermi approximation (which coincides with RPA only when $q<2 k_{\mathrm{F}}$ ). This hint is supported by the experimental curves which show a density-dependent enhancement of the drag ranging from a factor of 2 for the highest density, up to 10 for the lowest one and also from the peak at $q=2 k_{\mathrm{F}}$ of the drag intensity. It was also reported that the experimental data suggest a $N^{-4}$ behavior of $\rho_{\mathrm{D}}$ as a function of density which contradicts the $N^{-3}$ dependence predicted by the simplest RPA.

Motivated by these recent experiments, the aim of this work is to demonstrate the importance of effective intra- and inter-layer interactions in the observed drag resistivity. We consider several theoretical models to show the sensitivity of the drag resistivity to the strength of intra- and inter-layer correlations. As noted previously the RPA is not capable of predicting the quantitative behavior of drag resistivity, because it does not include the correlation effects. Our calculations show that the STLS theory does not account for the experimentally observed drag resistivity either because the correlation effects are overestimated. We employ the effective interaction theory of Vignale and Singwi [13] to model the inter-layer interaction in a double-quantum well system. We compare our theoretical calculations with the recent experimental results of Kellogg et al. [10] to find very good agreement. As the recent drag experiments on hole systems [11] were analyzed by Hwang et al. [12] we concentrate here on the electron double-layer systems. In the following we present the theoretical framework in which we perform our calculations and the results we obtain.

We consider two identical infinite layers of electrons separated by a distance $d$ such that there is no tunneling between them. Each layer is characterized by the dimensionless coupling constant $r_{\mathrm{s}}=a / a_{\mathrm{B}}$ where $a=1 / \sqrt{n \pi}$ is the average spacing between the electrons defined in terms of the area density $n$, and $a_{\mathrm{B}}=\hbar^{2} \varepsilon /\left(m^{*} e^{2}\right)$ is the effective Bohr radius, $\epsilon$ and $m^{*}$ being the background dielectric constant and electron effective mass. The experimental results reported by Kellogg et al. [10] used a sample with electron density $n=1.7-5.2 \times 10^{10} \mathrm{~cm}^{-2}$, corresponding to $r_{\mathrm{s}}$ values of 2.5-4.3. The bare Coulomb interaction between electrons on the same layer and on different layers is given as $V_{11}(q)=2 \pi e^{2} /(\epsilon q) F_{11}(q L)$ and $V_{12}=2$ $\pi e^{2} /(\epsilon q) e^{-q d} F_{12}(q L)$, respectively. $F_{11}$ and $F_{12}$ are infinite quantum-well form factors taking the finite width effects into account ( $L$ is the quantum-well width).

The drag resistivity $\rho_{\mathrm{D}}$ of a double-layer electron system with equal layer densities has been obtained in a variety of theoretical models. These include the Boltzmann equation [14], the memory function formalism [6], and diagrammatic perturbation theory [15]. Theoretical considerations lead to the same expression for $\rho_{\mathrm{D}}$ in terms of the effective interlayer interaction. To the lowest order in the dynamically screened effective inter-layer interaction $W_{12}(q, \omega)$, and specializing to the case of equal densities in the layers, $\rho_{\mathrm{D}}$ is given as $[6,9,14,15]$

$$
\begin{aligned}
\rho_{\mathrm{D}}= & \frac{\hbar^{2}}{8 n^{2} e^{2} k_{\mathrm{B}} T \pi^{2}} \int_{0}^{\infty} \mathrm{d} q q^{3} \int_{0}^{\infty} \\
& \mathrm{d} \omega\left|\frac{W_{12}(q, \omega ; T) \operatorname{Im} \chi_{0}(q, \omega ; T)}{\sinh \left(\hbar \omega / 2 k_{\mathrm{B}} T\right)}\right|^{2}
\end{aligned}
$$

where $\chi_{0}(q, \omega)$ is the density-density response function of a non-interacting single layer.

The key quantity in the above expression is the effective interlayer interaction $W_{12}$, for which the simplest approximation would be the bare inter-layer Coulomb interaction $V_{12}(q)$. The earlier calculations considered the statically screened Coulomb interaction within the RPA, which reads $W_{12}=V_{12} / \varepsilon(q)$ where the screening function is the one 
appropriate for a double-layer system $\varepsilon(q)=[1-$ $\left.V_{11} \chi(q, 0)\right]^{2}-\left[V_{12} \chi(q, 0)\right]^{2}$. The next level of approximation is to include the frequency dependent response functions in $\varepsilon(q, \omega)$ to obtain the dynamically screened RPA interaction.

In the previous drag experiments $[3,4]$ on electronelectron double-layer systems the density parameter was of the order of $r_{\mathrm{s}} \sim 1-2$, justifying the use of the RPA for theoretical modeling. The recent experiments of Kellogg et al. [10] on the other hand, are in the range of $r_{\mathrm{s}} \sim 2-4$ which are somewhat outside the area of applicability of RPA. Many-body effects beyond the RPA are treated within the self-consistent field approximation of Singwi et al. [8]. In particular, the corrections due to exchange and correlation to the effective interaction potentials associated with charge fluctuations are incorporated in this scheme in a physically motivated way. In this approach the dynamically screened inter-layer effective interaction is given by Świerkovski et al. [9] $W_{12}=$ $V_{12}\left(1-G_{12}\right) / \varepsilon(q, \omega)$, where $G_{11}$ and $G_{12}$ are intra- and inter-layer local-field factors, respectively.

In this work we use the formulation of effective twobody interactions in Coulomb liquids advanced by Vignale and Singwi [13]. This approach is the generalization to a two-component case of the screened interaction originally developed by Kukkonen and Overhauser [16]. In contrast to the RPA, which considers the screening of a test charge, the Kukkonen-Overhauser formulation takes into account of the medium. More recently, Richardson and Ashcroft [17] employed these effective interactions to explore the intrinsic superconductivity in a 3D electron-hole liquid. The Vignale-Singwi (VS) approach takes into account the direct interaction as well as the interactions stemming from the exchange of charge and spin fluctuations and it has the advantage of including direct and exchange contribution in a consistent manner. The Vignale-Singwi effective interaction is given by $[13,17]$

$W_{i j}(q, \omega)=V_{i j}(q)+\sum_{k l} \phi_{i k}(q, \omega) \chi_{k l}(q, \omega) \phi_{l j}(q, \omega)$,

where $\phi_{i j}=V_{i j}\left[1-G_{i j}\right]$ and $\chi$ is the density-density correlation function of the interacting system whose elements can be computed from $\left(\chi^{-1}\right)_{i j}=\delta_{i j} \chi_{0}^{-1}-\phi_{i j}$. More explicitly, the effective inter-layer interaction reads (we omit the $q$ and $\omega$-dependences for brevity)

$$
\begin{aligned}
W_{12}= & \frac{V_{12}\left(1-G_{12}\right)}{\left[1-V_{11}\left(1-G_{11}\right) \chi_{0}\right]^{2}-\left[V_{12}\left(1-G_{12}\right) \chi_{0}\right]^{2}} \\
& +V_{12} G_{12} .
\end{aligned}
$$

The form of $W_{12}$ within the Vignale-Singwi approach is similar to that in the STLS except for the last term $V_{12} G_{12}$. At a first glance this term seems to be small, but as will be shown, it gives a notable contribution to $\left|W_{12}\right|^{2}$. We note that the VS form of $W_{12}$ reduces to the RPA if the local-field factors are omitted.

In our calculations to be presented below, we have found that the STLS scheme produces a drag resistivity much higher than the experimental results. The Vignale-Singwi form of $W_{12}$ constructed via the STLS local-field factors also yields a large $\rho_{\mathrm{D}}$. The main reason for this is that the intralayer local-field factor $G_{11}$ is too large. In the previous applications of VS formalism parameterized forms satisfying certain limiting conditions of the local-field factors have been used. In the absence of reliable and accurate information on the local-field factors of a double-layer system, we take a phenomenological point of view. We have used the Hubbard approximation for the intra-layer localfield factor which has been known to yield good agreement with experimental results [18]. The Hubbard approximation for the intra-layer local-field factor is calculated as

$G_{11}^{H}(q, T)=-\frac{1}{n} \int \frac{\mathrm{d} \mathbf{k}}{(2 \pi)^{2}} \frac{\mathbf{q} \cdot \mathbf{k}}{q^{2}} \frac{V(k)}{V(q)}\left[S_{0}(\mathbf{q}-\mathbf{k} ; T)-1\right]$,

in which $S_{0}(q ; T)$ is the temperature dependent static structure factor of a non-interacting system. To include the correlation effects between the layers, we adopt the approach used by Dong and Lei [19] to calculate $G_{12}$. Here, the inter-layer local-field factor is assumed to have the form $G_{12}(q)=a q / \sqrt{q^{2}+b^{2}}$, and the parameters $a$ and $b$ are determined from the large and small $q$ limits. We call this the hybrid Hubbard-STLS approach.

We now present our calculations for drag resistivity $\rho_{\mathrm{D}}(T)$ using the theoretical models described above and compare them with the recent experimental results of Kellogg et al. [10]. The drag measurements are done on a double quantum-well of widths $L=180 \AA$ separated by a distance $d=280 \AA$. We therefore, model the finite width effects by an infinite square well which modifies the bare Coulomb interactions by a form factor, viz. $V_{i j}(q) \rightarrow V_{i j}(q) F_{i j}(q L)$. Although our calculations can easily be extended to double quantum wells of unmatched densities, in this work we consider only the case of equal density systems.

In Fig. 1 we show the calculated drag resistivity as a function of temperature in various theoretical models of effective inter-layer interaction and compare them with the experimental results of Kellogg et al. [10]. At a layer density of $n=2.3 \times 10^{10} \mathrm{~cm}^{-2}$ (corresponding to $r_{\mathrm{s}}=3.5$ ) we observe that the static RPA yields a very poor agreement with experiment as already noted by Kellogg et al. [10]. When the frequency dependence of the screening function $\varepsilon(q, \omega)$ appearing in the effective interaction $W_{12}$ is included the agreement with experimental data is somewhat improved. On the other hand, when the correlation effects beyond the RPA are incorporated within the STLS scheme we find that the calculated $\rho_{\mathrm{D}}$ lies much above the experimental results. We note that the intra- and interlayer local field factors $G_{i j}(q)$ we use in the STLS approach are independent of temperature. Note that the net effect of temperature dependent local-field factors is to reduce the drag resistivity. Nevertheless, the effects of temperature on $G_{i j}(q)$ are estimated to be much smaller than the difference between the calculated and measured $\rho_{\mathrm{D}}$. Finally, our 


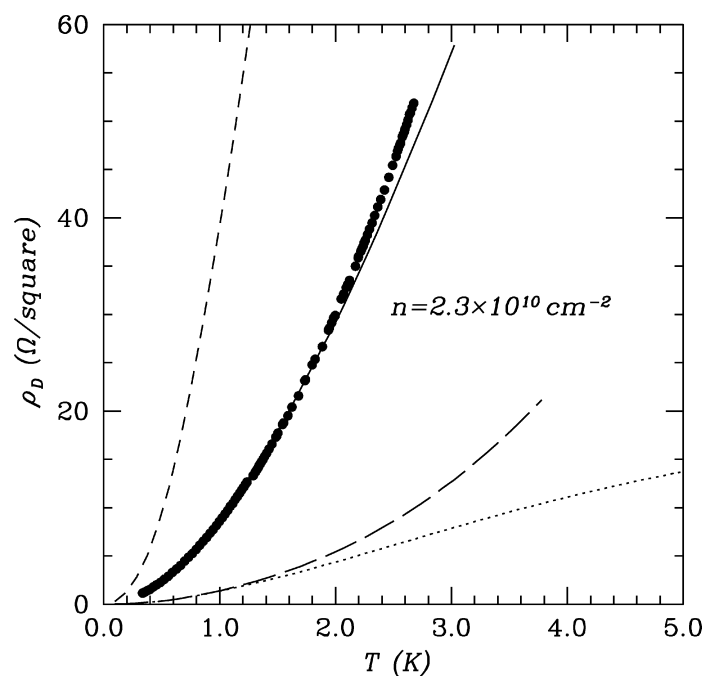

Fig. 1. The drug resistivity $\rho_{\mathrm{D}}$ as a function of temperature for matched layer densities $n=2.3 \times 10^{10} \mathrm{~cm}^{-2}$. The solid, shortdashed, long-dashed, and dotted lines represent calculations within the present model, STLS, dynamic-RPA, and static-RPA, respectively. The solid circles are the experimental data of Ref. 10.

present model of hybrid Hubbard and STLS approximations within the VS approach yields a very good quantitative agreement with the experimental data.

To further show the differences in various theoretical models we show in Fig. 2 the drag resistivity at $n=3.1 \times 10^{10} \mathrm{~cm}^{-2}$. When we use the STLS local-field

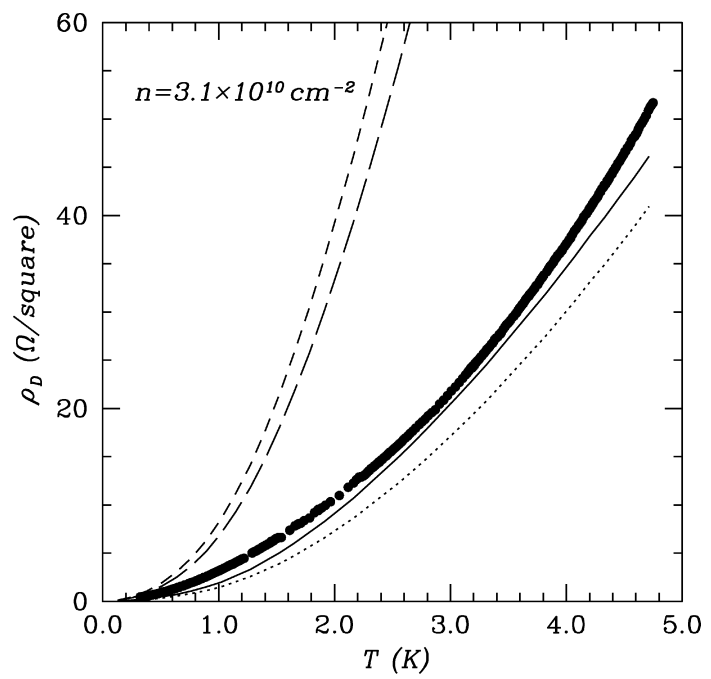

Fig. 2. The drug resistivity $\rho_{\mathrm{D}}$ as a function of temperature for matched layer densities $n=3.1 \times 10^{10} \mathrm{~cm}^{-2}$. The solid line shows $\rho_{\mathrm{D}}$ within the present model of VS approximation (using HubbardSTLS local-field factors). The dotted line uses the Hubbard approximation for $G_{11}$ and $G_{12}=0$. The long- and short-dashed lines represent calculations within the STLS and VS (including STLS local-field factors), respectively. The solid circles are the experimental data of Ref. 10. factors within the VS approach, $\rho_{\mathrm{D}}$ comes out to be even more enhanced than that in the STLS approximation. This, however, does not represent the experimental results of Kellogg et al. [10] well. It is also seen that the extra term $V_{12} G_{12}$ in the effective inter-layer interaction $W_{12}$ makes a significant contribution to $\rho_{\mathrm{D}}$. We find similar behavior of various theoretical models for $W_{12}$ at other densities for which Kellogg et al. [10] data are available (not shown in this work). It was also found by Hwang et al. [12] in their analysis of hole drag experiments of Pillarisetty et al. [11] the Hubbard local-field factors significantly enhance the drag resistivity. These comparisons clearly indicate the crucial role played by the effective inter-layer interaction $W_{12}$ on the drag resistivity $\rho_{\mathrm{D}}$. In particular, the intra-layer correlations as described by the STLS approach overestimate the drag resistivity.

The sensitivity of the drag resistivity to the model of the effective inter-layer interaction $W_{12}$, may also be seen in the $\log -\log$ plot of $\rho_{\mathrm{D}}$ as a function of the layer density shown in Fig. 3. As an illustration, at $T=4$, we find that the dynamical RPA fails to predict the density dependence of the observed $\rho_{\mathrm{D}}$ by systematically underestimating it. The static RPA screening essentially gives the same result as dynamic RPA except at very low densities. The STLS approximation which is supposed to describe the short-range correlation effects better than RPA also shows significant differences in the regime of densities probed by the Kellogg et al. [10] experiment. Our present approximation which treats the intra-layer correlation effects at the Hubbard approximation level provides an overall satisfactory agreement with the experimental data. Another useful information we draw from these plots is that the density dependence of $\rho_{\mathrm{D}}$ is essentially

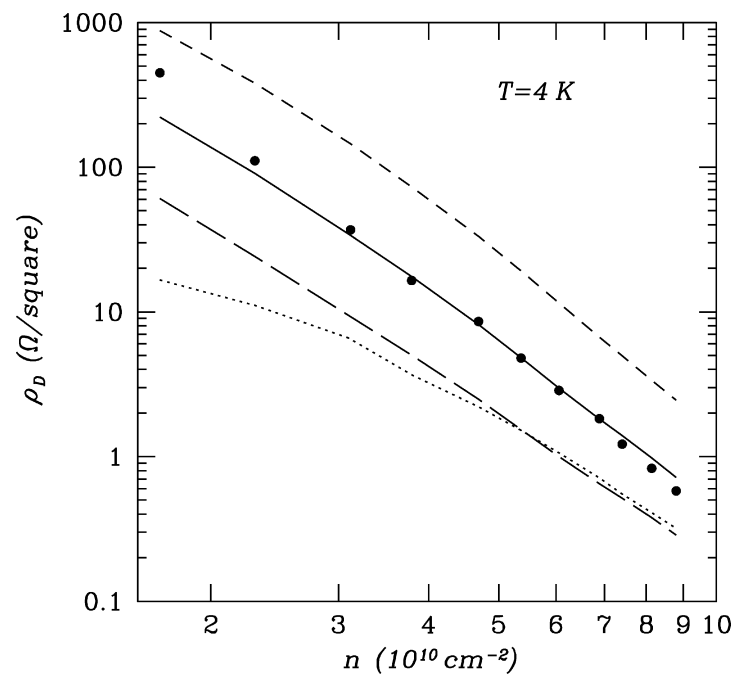

Fig. 3. The drug resistivity $\rho_{\mathrm{D}}$ as a function of the layer density for $T=4 \mathrm{~K}$. The solid, short-dashed, long-dashed, and dotted lines represent calculations within the present model, STLS, dynamicRPA, and static-RPA, respectively. The solid circles are the experimental data of Ref. 10. 


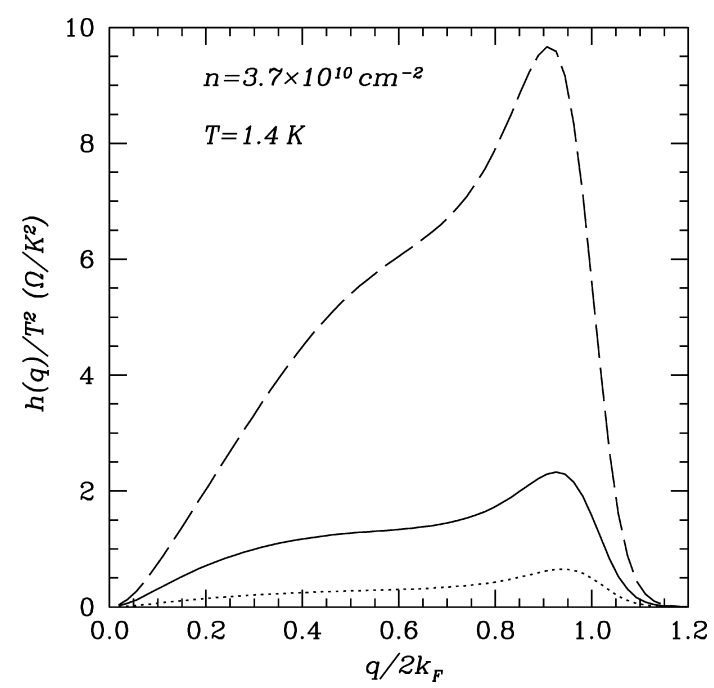

Fig. 4. The drug intensity $h(q)$ divided by $T^{2}$ as a function of momentum $q$ at $T=1.4 \mathrm{~K}$ and $n=3.7 \times 10^{10} \mathrm{~cm}^{-2}$. The solid, dashed, and dotted lines represent calculations within the present model, STLS, and dynamic-RPA, respectively.

similar for dynamic RPA, STLS, and the present model. They all roughly follow the $n^{-4}$ rule, in contrast to the prediction of $n^{-3}$ of the simple model $[3,6]$.

The last point we consider is the contribution of the $2 k_{\mathrm{F}}$ electron-electron scattering processes. The possibility of these processes is experimentally confirmed by the behavior of the drag intensity $h(q, T)$ defined by $\rho_{\mathrm{D}}(T)=\int \mathrm{d} q h(q, T)$. We show the calculated $h(q, T) / T^{2} s$ in various approximations as a function of $q / 2 k_{\mathrm{F}}$ in Fig. 4 . At low temperatures it has a peak around $q=2 k_{\mathrm{F}}$, which shifts towards lower momenta when the temperature increases. The magnitude of $h(q, T)$ however, depends very sensitively on the form of the effective interaction $W_{12}$ as Fig. 4 indicates. As the previous figures suggest the VS approach with the local-field factors calculated in a hybrid Hubbard-STLS approximation yields the best agreement with experimental results.

In summary, we have performed calculations of the drag resistivity in connection with the recent experiments of Kellogg et al. [10] at low density. We have shown that the observed drag resistivity depends very sensitively on the effective inter-layer interaction $W_{12}$. The static and dynamic screening effects incorporated at the level of RPA underestimate the measured drag resistivity. The STLS approximation on the other hand overestimates it. A theoretical model based on the formulation of Vignale and Singwi [15] describing the intra-layer correlations via a local-field factor within the Hubbard approximation yields very good quantitative agreement with the experimental data. Also important is to include the inter-layer correlations through the local-field factor $G_{12}$. In particular, the temperature dependence of $\rho_{\mathrm{D}}$ at fixed layer densities and density dependence at fixed temperatures within the VS approach is accurately reproduced. Further theoretical work is necessary to improve on the local-field factors that enter the VS effective interaction. Comparison with other available drag data for instance in the plasmon region would be the subject of future work.

\section{Acknowledgements}

This work was partially supported by TUBITAK under Grant No. TBAG-2005, by NATO under Grant No. SfP971970, by the Turkish Academy of Sciences (TUBA), and by the Turkish Department of Defense under Grant No. KOBRA-001. V. M. acknowledges support from NATOTUBITAK and the Romanian Ministry of Education and Research under CERES contract No.6. We thank Professor J. P. Eisenstein and Dr M. Kellogg for providing us with the digitized form of their data.

\section{References}

[1] T. Ando, A.B. Fowler, F. Stern, Rev. Mod. Phys. 54 (1982) 437. C.B. Hanna, D. Haas, J.C. Díaz-Vélez, Phys. Rev. B 61 (2000) 13-882.

[2] M.B. Pogrebinskii, Sov. Phys. Semicond. 11 (1977) 372. P.J. Price, Physica B 117B (1983) 750.

[3] T.J. Gramila, J.P. Eisenstein, A.H. MacDonald, L.N. Pfeiffer, K.W. West, Phys. Rev. Lett. 66 (1991) 1216. T.J. Gramila, J.P. Eisenstein, A.H. MacDonald, L.N. Pfeiffer, K.W. West, Phys. Rev. B 47 (1993) 12957.

[4] For a review see.A.G. Rojo, J. Phys.: Condens. Matter 11 (1999) R31.

[5] N.P.R. Hill, J.T. Nichols, E.H. Linfield, M. Pepper, D.A. Ritchie, G.A.C. Jones, B.Y.-K. Hu, K. Flensberg, Phys. Rev. Lett. 78 (1997) 2204.

[6] L. Zheng, A.H. MacDonald, Phys. Rev. B 49 (1994) 5522. A.P. Jauho, H. Smith, Phys. Rev. B 46 (1993) 4420.

[7] H. Noh, S. Zelakiewicz, X.G. Feng, T.J. Gramila, L.N. Pfeiffer, K.W. West, Phys. Rev. B 58 (1998) 12621.

[8] K.S. Singwi, M.P. Tosi, R.H. Land, A. Sj, ölander, Phys. Rev. 176 (1968) 589.

[9] L. Świerkovski, J. Szymaǹski, Z.W. Gortel, Phys. Rev. B 55 (1997) 2280.

[10] M. Kellogg, J.P. Eisenstein, L.N. Pfeiffer, K.W. West, Solid State Commun. 123 (2002) 515.

[11] R. Pillarisetty, H. Noh, D.C. Tsui, E.P. De Poortere, E. Tutuc, M. Shayegan, Phys. Rev. Lett. 89 (2002) 016805.

[12] E.H. Hwang, S. Das Sarma, V. Braude, A. Stern, cond-mat/ 0202249

[13] G. Vignale, K.S. Singwi, Phys. Rev. B 31 (1985) 2729. G. Vignale, K.S. Singwi, Phys. Rev. B 32 (1985) 2156.

[14] K. Flensberg, B.Y.-K. Hu, A.P. Jauho, J.M. Kinaret, Phys. Rev. B 52 (1995) 14761.

[15] A. Kamenev, Y. Oreg, Phys. Rev. B 52 (1995) 7516.

[16] C.A. Kukkonen, A.W. Overhauser, Phys. Rev. B 20 (1979) 550.

[17] C.F. Richardson, N.W. Aschroft, Phys. Rev. B 52 (1997) 15130.

[18] K. Flensberg, B.Y.-K. Hu, Phys. Rev. Lett. 73 (1994) 3572. K. Flensberg, B.Y.-K. Hu, Phys. Rev. B 52 (1995) 14796.

[19] B. Dong, X.L. Lei, J. Phys.: Condens. Matter 10 (1998) 7535. 